\title{
Ultrasonography-guided botulinum toxin injection to the cricopharyngeus muscle: a case report and technical notes
}

\author{
Yi-Chian Wang ${ }^{1,2}$, Chueh-Hung Wu${ }^{1}$, Shaw-Gang Shyu ${ }^{1}$, Ming-Yen Hsiao ${ }^{1}$, Tyng-Guey \\ Wang ${ }^{1}$
}

${ }^{1}$ Department of Physical Medicine and Rehabilitation, National Taiwan University Hospital, College of Medicine, National Taiwan University, ${ }^{2}$ Supreme Rehabilitation Clinic, Taipei, Taiwan

\begin{abstract}
Dysphagia associated with the cricopharyngeus muscle $(\mathrm{CPm})$ dysfunction negatively influences the quality of life. This high-pressure region must relax and the lumen must open for smooth food passage. The CP muscle is therefore a common target of chemodenervation with botulinum toxin (BTX). Here we presented a patient with severe left lateral medullary syndrome and non-relaxation of the $\mathrm{CPm}$. We described how to localize the $\mathrm{CPm}$ in the transverse and longitudinal views under ultrasonography and offered a video demonstrating ultrasonography-guided BTX injection. Ultrasonography-guided CPm injection with BTX may serve as a reliable, rapid, and effective choice for treatment of cricopharyngeal dysphagia.
\end{abstract}

Keywords: dysphagia; ultrasound; botulinum toxin; cricopharyngeus; stroke

\section{Introduction}

Cricopharyngeus muscle (CPm) dysfunction leads to dysphagia and negatively influences the quality of life. The $\mathrm{CPm}$ is therefore a common target of chemodenervation with botulinum toxin (BTX). The techniques for administration of BTX to the CPm include CT-guided in-

Received 07.08.2019 Accepted 15.10.2019

Med Ultrason

2019, Vol. 22, No 3, 370-373

Corresponding author: Chueh-Hung Wu

Departments of Physical Medicine and

Rehabilitation, National Taiwan University

Hospital, College of Medicine,

National Taiwan University, Taipei, Taiwan

No.7, Zhongshan S. Rd., Zhongzheng Dist.,

Taipei City 100, Taiwan

Phone: 886-2-23123456 ext.66473

E-mail: b88401062@ntu.edu.tw jection [1], endoscopic injection under general anesthesia or local anesthesia [2-4], and percutaneous injection with electromyographic guidance $[5,6]$. In this case report, we present the technique for ultrasonography-guided BTX injection in a patient with severe left lateral medullary syndrome and non-relaxation of the CPm.

\section{Case report}

A 68-year-old man, with left lateral medullary syndrome and severe dysphagia, had an indwelling nasogastric tube because he refused a gastrostomy. His speech pathologist kept treating him regularly but very little progress was obtained. When he was referred to our research team, he still needed to spit out his saliva persistently, heaping up to about $1000 \mathrm{~cm}^{3}$ per day. In addition, he experienced severe choking and coughing at night. A video fluoroscopic swallow study showed a large amount 


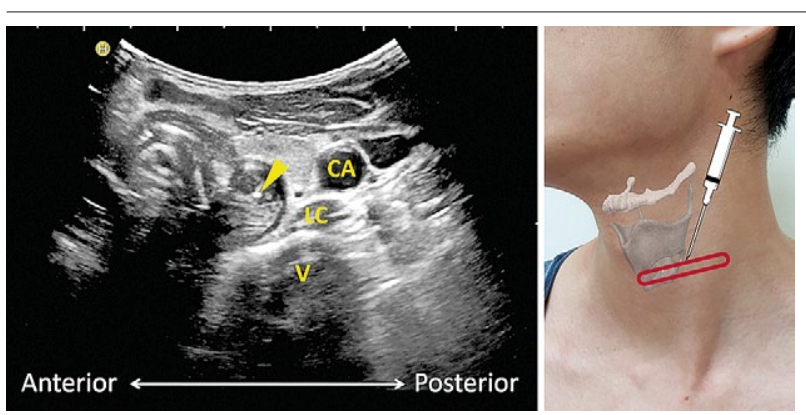

Fig 1. The cricopharyngeal muscle $(\mathrm{CPm})$ injection was performed with real-time ultrasound guidance with an electromyographic needle via transverse view and out-of-plane approach. The needle tip (arrow head) emerged within the CPm. CA: carotid artery. LC: longus colli muscle. V: vertebra.

of aspiration into trachea, severe hypo-contractility of middle and inferior pharyngeal constrictor and non-relaxation of $\mathrm{CPm}$. After a thorough discussion, the patient received 50 U BTX (BOTOX ${ }^{\circledR}$, Allergan) injections. The $\mathrm{CPm}$ injection can be performed safely under real-time ultrasound guidance with an electromyographic needle (fig 1 and Supplementary video, on the journal site).

Because a longitudinal, in-plane approach, might be impeded by thyroid cartilage calcification, the transverse, out-of-plane approach can be performed with better reliability. The CPm was identified using a (Hitachi Aloka) ultrasound machine and a high-resolution linear probe (18-5L). The patient lay supine with head rotated to the right side. While the probe was placed at position A (fig $2 \mathrm{~A}$ ), the thyroid cartilage appeared as a hypoechoic, homogenous plate, sometimes with interspersed calcification spots in elderly subjects. Deep to the thyroid cartilage, the arytenoid bone connects and controls the vocal cord when phonating. The hypopharynx sits anterior to the longus colli muscle, lined by hyperechoic mucous membrane internally and surrounded by the hypoechoic muscular rim externally. During a normal swallowing process, hyperechoic water droplets or food content passes through the hypopharynx while the vocal cord area remains clear. When severe aspiration happens, hyperechoic water could be observed passing between the vocal cords.

When the probe was moved to position B (fig 2B), the thyroid cartilage gradually tapered, and the cricoid cartilage appeared deep to the cornu of thyroid cartilage. The $\mathrm{CPm}$ is an oval-shaped, circular structure. Since the CPm contracts persistently at rest, the typical 5-layer digestive tube structure cannot be discerned. Instead, the hyperechoic mucosal membranous lining forms a bright, irregular center. During a normal swallowing process, hyperechoic water or food passes through the dotted circle and the $\mathrm{CPm}$ opens and closes up quickly. At this level, the needle is inserted proximally to the probe, and just laterally to the border of thyroid cartilage. As the needle was advanced caudally, the needle tip emerged as indicated by the arrow head in figure 1. BTX was then delivered smoothly into the anterior and posterior wall of $\mathrm{CPm}$.

While the probe was moved to position C (fig 2C), the cricoid cartilage gradually tapered, and the tracheal cartilage appeared. The upper esophagus was a spindle-contour tube structure. The tube wall is composed of a typical 5-layer structure, sequentially as hyperechoic superficial mucosa, hypoechoic deep mucosa, hyperechoic submucosa, hypoechoic muscular rim, and hyperechoic serosa. When seeing this structure, the probe should be moved slightly proximal to identify the $\mathrm{CPm}$ level. During a normal swallowing process, the hyperechoic water or food passes inside the flaccid esophageal tube. Briefly after the
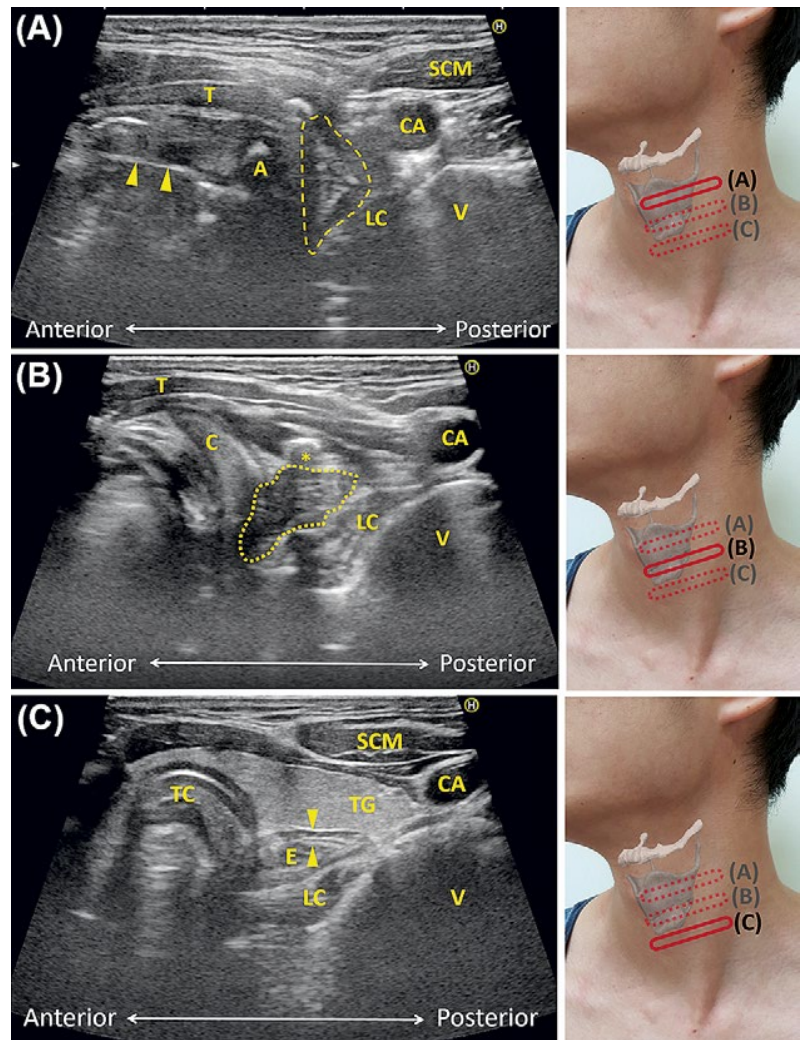

Fig 2. Serial scanning of cricopharyngeal muscle (CPm) with transverse view. Ultrasonography images were shown at the corresponding levels of scanning. Dotted circles indicated hypopharynx and $\mathrm{CPm}$ in figure $2 \mathrm{~A}$ and $2 \mathrm{~B}$, respectively. The upper esophagus (E) was a spindle-contour tube structure, composed of typical 5-layer structure (between arrowheads in figure 3C). T: thyroid cartilage; A: arytenoid; arrow heads: vocal cord; LC: longus colli muscle; CA: carotid artery; SCM: sternocleidomastoid muscle; V: vertebra; C: cricoid cartilage; *: cornu of thyroid cartilage; TC: tracheal cartilage. Arrow heads in figure $2 \mathrm{~A}$ : vocal cord. 
water passage, the esophageal muscular wall contracts and rounds up for 1-2 seconds conspicuously and then relaxes, which indicates a reflexive post-swallow peristalsis activity.

Serial scanning of $\mathrm{CPm}$ with longitudinal view (fig 3 ) can be also performed if the level of the vertebra needs to be clarified, with the patient in the same position. As the probe was placed at position A (fig 3A), the thyroid cartilage appeared as a thin, homogenous plaque, and the cross-section view of the vocal cord appeared deep into it. The cricoid cartilage manifested as an oval shape structure distal to the thyroid cartilage. Both cartilages were lined by a thin layer of neck strap muscles, including the sternothyoid, sternohyoid, and thyrohyoid muscles. As the probe moved to position B (fig 3B), the hypopharynx appeared as a wide, tube-like structure behind the thyroid cartilage, with heteroechoic content inside. The $\mathrm{CPm}$ emerges behind the cricoid cartilage, connecting the esophagus and hypopharynx. The food tube is continuous, with no clear-cut border between the hypopharynx, $\mathrm{CPm}$, and esophagus. An electromyographic needle can be used to identify the specific segment with persistently high muscle activities, indicating the $\mathrm{CPm}$. Thyroid cartilage calcification is common in elderly people, and the hypopharynx might be shaded and can not be revealed by ultrasound (fig 3C).

Two months after injection, the amount of spit saliva decreased to $300 \sim 500 \mathrm{cc}$ per day, and he had less choking and coughing during sleep. His breathing sound also became clear. He kept swallow therapy for 3 more months, and the condition was stable. However, he still could not try other food intake. The patient refused a $2^{\text {nd }}$ injection due to financial concerns.

\section{Discussion}

Dysphagia associated with $\mathrm{CPm}$ dysfunction would negatively influence patient quality of life. $\mathrm{CPm}$ is located at the junction between the pharynx and the esophagus. This high-pressure region must relax and the lumen must open for smooth food passage. The CPm is therefore a common target of interventions for dysphagia, including balloon dilation, myotomy and chemodenervation with BTX $[7,8]$. Recently, ultrasonography-guided injection is attracting more attention because of several advantages such as free from radiation exposure, sparing general or local anesthesia, and real-time visualization of the neurovascular anatomy, thus minimizing the risk of unintentional injury to these vital structures [9].

In this report, we described how to localize the $\mathrm{CPm}$ in the transverse and longitudinal views under ultrasonography. In a previous report [9], a patient with active
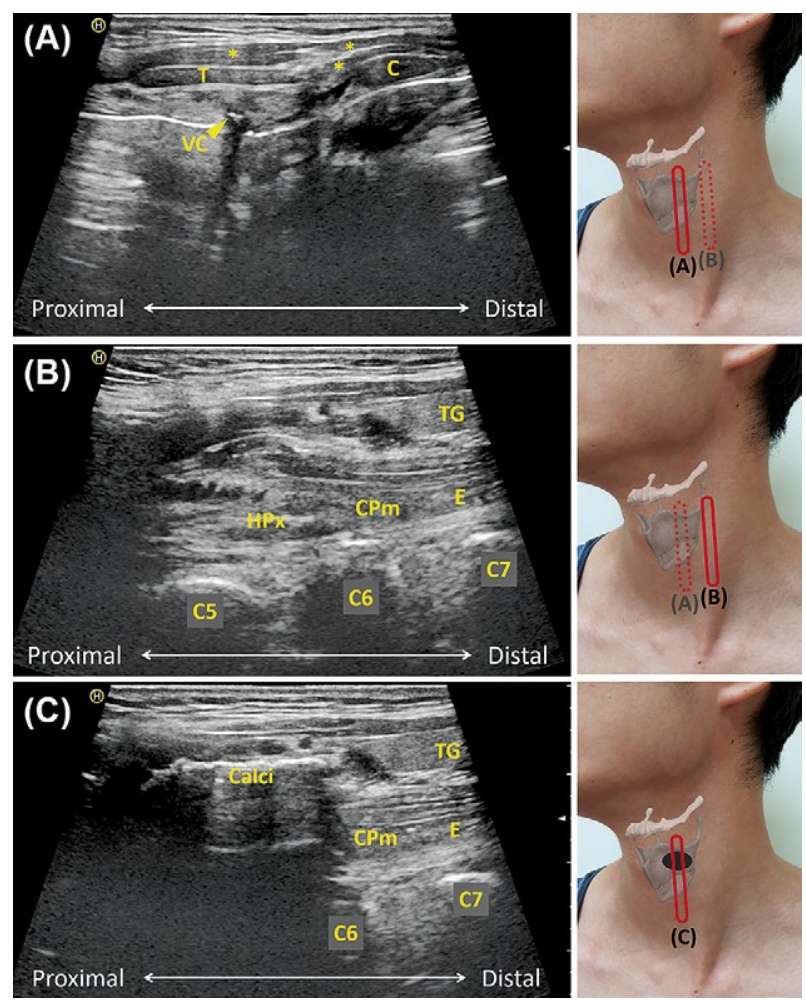

Fig 3. Serial scanning of cricopharyngeal muscle $(\mathrm{CPm})$ with longitudinal view. T: thyroid cartilage; VC: vocal cord; C: cricoid cartilage; *: neck strap muscles; HPx: hypopharynx; E: esophagus; TG: thyroid gland; C5: the fifth vertebra; C6: the sixth vertebra; C7: the seventh vertebra; Calci: calcification in the thyroid cartilage.

pharyngeal constrictors and tight $\mathrm{CPm}$ responded well to BTX injection. However, in this patient, who had hypoactive pharyngeal constrictors and tight $\mathrm{CPm}$, BTX injection into $\mathrm{CPm}$ only facilitated saliva leakage through a cricopharyngeal opening to reduce the amount of saliva aspiration. To initiate a successful swallow, strength from tongue, oral base muscles and pharyngeal constrictors are necessary. For patients with flaccid oral base and pharyngeal muscles, BTX injection in to CPm should be implemented with other strategies to increase laryngeal elevation, such as Masako maneuver, Mendelsohn maneuver, Shaker exercise, resisted jaw opening, electrical stimulation, or hyoid sling surgery.

In conclusion, the procedure did help this patient make some progress and reduced the severity of dysphagia, but further research is required to validate the efficacy of CPm injection with BTX.

\section{References}

1. Atkinson SI, Rees J. Botulinum toxin for cricopharyngeal dysphagia: case reports of CT-guided injection. J Otolaryngol 1997;26:273-276. 
2. Haapaniemi JJ, Laurikainen EA, Pulkkinen J, Marttila RJ Botulinum toxin in the treatment of cricopharyngeal dysphagia. Dysphagia 2001;16:171-175.

3. Shaw GY, Searl JP. Botulinum toxin treatment for cricopharyngeal dysfunction. Dysphagia 2001;16:161167.

4. Jeong SH, Kim YJ, Kim YJ, et al. Endoscopic botulinum toxin injection for treatment of pharyngeal dysphagia in patients with cricopharyngeal dysfunction. Scand J Gastroenterol 2018;53:1201-1205.

5. Alfonsi E, Merlo IM, Ponzio M, et al. An electrophysiological approach to the diagnosis of neurogenic dysphagia: implications for botulinum toxin treatment. J Neurol Neurosurg Psychiatry 2010;81:54-60.
6. Kim MS, Kim GW, Rho YS, Kwon KH, Chung EJ. Officebased Electromyography-guided Botulinum Toxin Injection totheCricopharyngeusMuscle:OptimalPatientSelectionand Technique. Ann Otol Rhinol Laryngol 2017;126:349-356.

7. Kelly EA, Koszewski IJ, Jaradeh SS, Merati AL, Blumin $\mathrm{JH}$, Bock JM. Botulinum toxin injection for the treatment of upper esophageal sphincter dysfunction. Ann Otol Rhinol Laryngol 2013;122:100-108.

8. Terre R, Panades A, Mearin F. Botulinum toxin treatment for oropharyngeal dysphagia in patients with stroke. Neurogastroenterol Motil 2013;25:896-e702.

9. Wang YC, Shyu SG, Wu CH, Wang TG. Ultrasound-Guided Injection of Botulinum Toxin for Cricopharyngeal Dysphagia. Am J Phys Med Rehabil 2018,97:e102-e103. 